\title{
Micro-channel cooling in high energy physics
}

\author{
M. Vos* \\ IFIC (UVEG/CSIC) Valencia, C./ Catedratico Jose Beltran 2, 46980 Paterna, Spain \\ E-mail: marcel.vos@ific.uv.es
}

\begin{abstract}
With the development of thinner and more precise silicon detectors for vertexing and tracking in collider experiments, the design of sufficiently thin and stable supports and services is increasingly challenging. In this contribution an overview is given of the deployment of micro-channel cooling in high energy physics. The emphasis is on a recently proposed approach to integrate the cooling channels in the silicon sensor and the connectivity of the micro-channel circuit to the overall system. Measurements and finite-element simulations of prototype assemblies are presented. The cooling performance is found to exceed that of more traditional systems by a large factor. The impact of micro-channel cooling on the mechanical stability is also discussed.
\end{abstract}

The 25th International workshop on vertex detectors

September 26-30, 2016

La Biodola, Isola d'Elba, ITALY

${ }^{*}$ Speaker. 


\section{Introduction}

Silicon vertex detectors and trackers are the most granular elements of collider experiments, with read-out channel density reaching $\mathscr{O}\left(10^{4}\right)$ channels $/ \mathrm{cm}^{2}$ in modern pixel detectors. The power dissipated in the Front End electronics, and the sensor itself, must be removed from the detector, maintaining a low, stable, and uniform temperature throughout the tracker. In environments with high non-ionizing radiation doses, i.e. operation in the high-luminosity phase of the LHC, sensors must be operated at temperatures well below $0^{\circ} \mathrm{C}$ (down to $-30^{\circ} \mathrm{C}$ ) to reduce the leakage current. The spatial resolution and the channel and power density are expected to further increase in future facilities, such as high-energy lepton colliders. At the same time the material budget reserved for supports and services decreases. The development of low-mass cooling options with a minimal impact on the mechanical stability of the detector therefore represents an important challenge.

Micro-channel cooling, with the coolant circulating through narrow channels etched in silicon, represents a powerful solution. A schematic representation is shown in the cartoon in Figure 1. Silicon cooling plates with an integrated cooling manifold offer competitive performance compared to more classical cooling systems in terms of the contribution to the material budget and the contact area with the coolant with a radiation. The use of the same material in the sensor and the cooling plate avoids stresses due to mismatches in thermal expansion coefficients.

The integration of cooling and the detector (sensor or read-out chips) can be taken one step further still. Embedding the cooling channels inside the silicon of the active element we remove the last thermal barrier in the design and minimize the temperature difference between heat source and coolant.

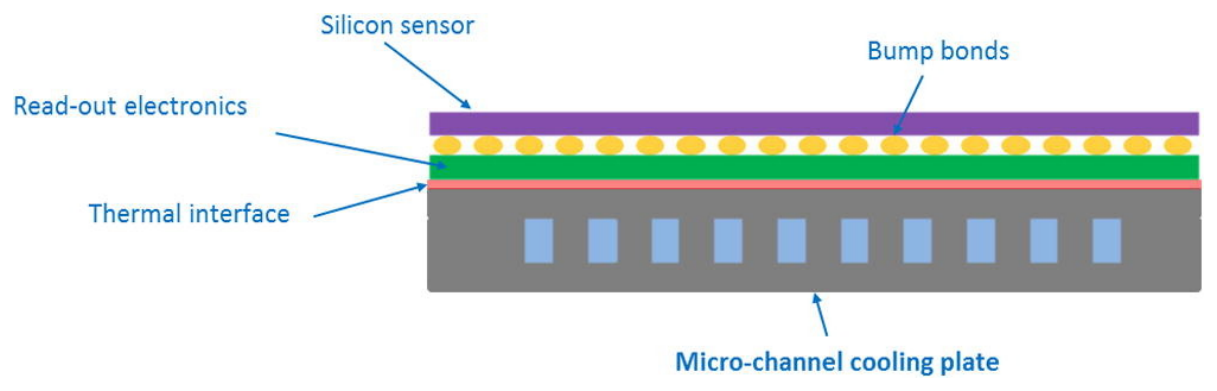

Figure 1: A schematic view of a possible use of micro-channels to cool a hybrid pixel detector. The upper (purple) layer represents the sensor. It is connected to the read-out electronics (green) through an array of solder connections realized in the flip-chip bump-bonding technique (orange). The typical bump pitch in hybrid pixel detectors is several tens to hundreds of $\mu \mathrm{m}$. The detector is cooled by establishing a thermal contact through a thin glue layer (pink) with a silicon cooling plate (dark grey). The plate is bonded or glued (violet layer) to a second silicon wafer equipped with a number of narrow cooling channels (light blue rectangles), through which the coolant circulates. The typical dimension of the channels in the applications discussed here is $100-300 \mu \mathrm{m}$. Figure from Ref. [1].

Section 2 presents a brief overview of existing efforts to deploy micro-channel cooling in high energy physics. In Section 3 the approaches adopted for creation of the manifold in the silicon and interconnect technology is discussed. Finally, Section 4 presents the recent development of a cooling solution where the micro-channels are integrated in the silicon sensors and conclusions are presented in Section 5. 


\section{Applications in high-energy physics experiments}

\subsection{NA62 GigaTracker}

NA62 [2], a rare kaon decay experiment in the CERN North Area, is the first high-energy physics experiment to use micro-channel cooling. The GigaTracker [3, 4] consists of three hybrid pixel detector planes installed along the beam line to measure the momentum, angle and time of incoming kaons before the decay. Each pixel module dissipates $48 \mathrm{~W}$ and is mounted on a silicon cooling plate[1,5] that covers the $3 \times 6 \mathrm{~cm}^{2}$ sensitive area (power density $2.6 \mathrm{~W} / \mathrm{cm}^{2}$ ). The plate hosts 150 cooling channels (width $\times$ height $=200 \times 70 \mu \mathrm{m}^{2}$ ). A photograph is shown in Figure 2 (a). In the acceptance the covers are as thin as $30 \mu \mathrm{m}$, reducing the total thickness of the plate to 150 $\mu \mathrm{m}$ and the average contribution to the material budget to $0.13 \% X_{0}$. Mono-phase perfluorocarbon $\mathrm{C} 6 \mathrm{~F} 14$ at a pressure of $3.5 \mathrm{bar}$ is used to cool the detector down to $-10^{\circ} \mathrm{C}$. Tests on prototypes show good uniformity, with a temperature gradient of $1^{\circ} \mathrm{C}$ over the sensor area.

The GigaTracker was installed and fully commissioned in 2014-2015. Problems with the thickness and uniformity of the initial production of cooling plates [6] were solved by procuring a new batch of cooling plates with a thickness of 210 or $270 \mu \mathrm{m}$. The uniformity of the thickness is improved by adding buried oxide layers that act as a stop for the deep reactive ion etching used to thin the samples. The integration of the micro-channel device with the detector module and electronic board required several precision jigs and the development of strict procedures. One of the stations failed due to a hydraulic shock, the result of an error in the control of the cooling plant. A bad thermal contact between the cooling plate and one of the read-out chips on station 2 could be repaired in situ. Despite these problems the NA62 experiment has successfully taken data in 2015 and 2016, with the GigaTracker operating at over 99\% efficiency.

\subsection{LHCb VELO upgrade}

The LHCb experiment will undergo an important upgrade during the second long shutdown (Long Shutdown 2, or LS2) of the Large Hadron Collider, scheduled for 2019-2020. The Vertex Locator (VELO) will be replaced by a hybrid pixel detector [7, 8]. The read-out module consisting of 4 sensors and twelve VeloPix ASICs has a total power consumption of approximately $45 \mathrm{~W}$ over a $24 \mathrm{~cm}^{2}$ area (power density $1.9 \mathrm{~W} / \mathrm{cm}^{2}$ ). The sensor must be cooled to $-20^{\circ} \mathrm{C}$ to cope with the non-ionizing radiation dose equivalent to $10^{16} 1 \mathrm{MeV}$ neutrons $/ \mathrm{cm}^{2}$ [7].

The central part of the module is formed by a $400 \mu \mathrm{m}$ thick silicon cold plate [9]. The heat generated by the read-out modules is removed by two-phase $\mathrm{CO}_{2}$ flowing through narrow channels in the plate (width $\times$ height $=200 \times 70 \mu \mathrm{m}^{2}$ ). A relatively thick $(140 \mu \mathrm{m})$ plate and cover are required to stand the high pressure (up to 60 bar in boundary conditions) of the evaporative $\mathrm{CO}_{2}$ cooling system. A further adaptation to evaporative cooling are structures to "boil" the $\mathrm{CO}_{2}$, shown in Figure 2 (c). The width of the cooling channels changes from 30 to $200 \mu \mathrm{m}$.

Simulations show that micro-channel cooling can efficiently remove the heat load, maintaining a small temperature difference with respect to the coolant temperature throughout the assembly, with a $5^{\circ} \mathrm{C}$ gradient over the $5 \mathrm{~mm}$ overhang closest to the interaction point, where the sensor extends beyond the cold plate to reduce the material. LHCb has tested prototypes, showing that key elements (cooling plate, connectors) can stand pressures well in excess of the requirement. 

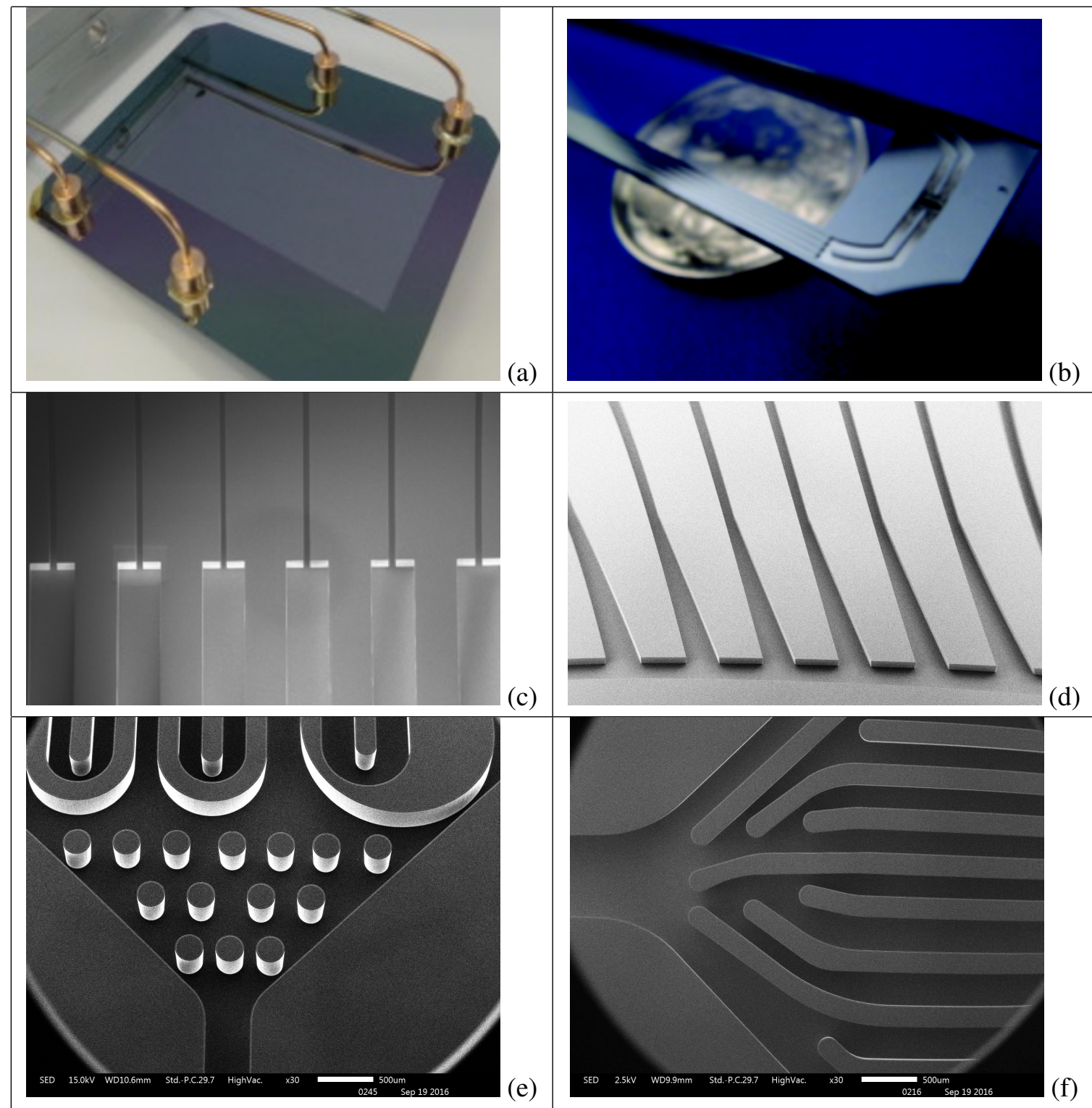

Figure 2: Photograph of the NA62 cooling plate [1] (a), photograph of a prototype structure for the ALICEITS [10] (b), X-ray image of the high-impedance, restricted-width structures where the liquid $\mathrm{CO}_{2}$ evaporates in the LHCb VELO cold plate [7] (c), electron microscopy image of the broadening channels produced by CNM [11] (d), X-ray images of the structures integrated in detector-grade silicon by HLL [12] (e,f).

\subsection{ALICE ITS upgrade}

The ALICE inner tracker based on Monolithic Active Pixel Sensors (MAPs) will be upgraded during LS2 [10, 13]. The ITS staves will have a length ranging from 27 to $147 \mathrm{~cm}$, with a power density of $300 \mathrm{~mW} / \mathrm{cm}^{2}\left(100 \mathrm{~mW} / \mathrm{cm}^{2}\right)$ in the inner (outer) layers. The detector is to be operated around room temperature. The baseline cooling solution relies on carbon fiber cold plate cooled by a water flow. Alternatives under consideration include a water flow through a polyimide structure with small $(0.8 \mathrm{~mm} \times 0.3 \mathrm{~mm})$ cooling channels and micro-channel cooling in silicon support structures [14]. 
The micro-channel option envisages two-phase $C_{4} F_{10}$ cooling in silicon structures with $100 \times$ $100 \mu \mathrm{m}$ channels [10]. A photograph of a prototype is shown in Figure 2 (b). The contribution to the material is reduced by removing the silicon in the central part of the cooling plates, leaving a support frame with integrated cooling. As the ITS staves are much longer than commercially available (8") wafers, several such structures must be daisy-chained. Preliminary tests with three cooling frames connected in parallel show that the entire stave can be efficiently cooled this way. The interconnects are within the acceptance of the experiment and a strict control of the material budget is mandatory.

\subsection{ATLAS upgrade}

The DESY and CNM groups involved in the ATLAS tracker upgrade for LS2 have explored micro-channel-based cooling as an alternative to the Titanium cooling pipes. The groups have designed a silicon cooling plate [11] the size of a 4" wafer with 60 cooling channels. The design has several innovative features, such as a varying width in the manifold to ensure uniform flow throughout the area (see Figure 2 (d)). Several prototype cooling plates were produced at CNM and tested with a mono-phase liquid flow. The hydrodynamic and cooling performance are predicted accurately by a Finite Element simulation [11].

\section{Micro-channel technology}

\subsection{Foundries}

The technology to produce micro-channel manifold is accessible in several commercial vendors. The structures used for the pioneering NA62 and LHCb VELO prototypes are designed at CERN and produced at CEA-LETI in Grenoble. The CERN group also has close links with EPFL Lausanne and the University of Southampton. Demonstrated in-house production capacity is available in a number of institutes with close ties to high energy physics, such as the Spanish national micro-electronics lab CNM-IMB in Barcelona, the Fondazione Bruno Kestler (FBK, for a description of their micro-channel cooling activities at FBK the reader is referred to Ref. [15]) in Trento and the semi-conductor laboratory (HLL Munich) of the Max Plank Society. The photograph and X-ray images of Figure $2(\mathrm{e}, \mathrm{f})$ are evidence of the precision and flexibility of silicon process techniques to produce structures of great complexity.

\subsection{Hydraulic connectors}

The experiments that have pioneered the development of micro-channel cooling solutions (NA62, LHCb) are characterized by their forward layout. Supports and services are largely located outside the acceptance of the experiment. The interconnects chosen by both experiments are robust out-of-plane Kovar connectors of the Figure 3 (a). Nickel/Cobalt pipes with a typical diameter of $1 \mathrm{~mm}$ are soldered to the silicon, yielding a solid connection that can stand a coolant pressure of 200 bar and a pull strength of $600 \mathrm{~N}$.

Applications in experiments with full solid angle $(4 \pi)$ coverage (ALICE-ITS, ATLAS/CMS, future lepton colliders), where the connectors are located in the acceptance, require a low-mass solution. Connectors in plastic, with a radiation length that is approximately 20 times longer than 


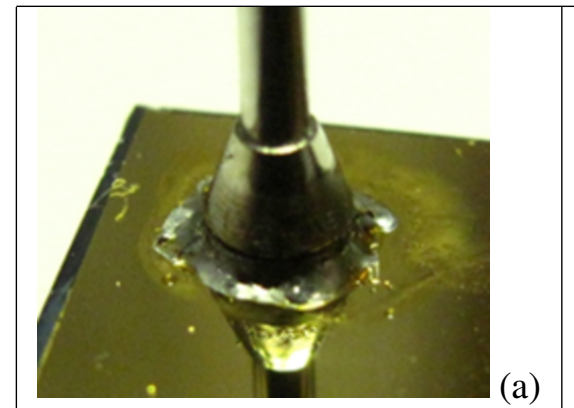

(a)

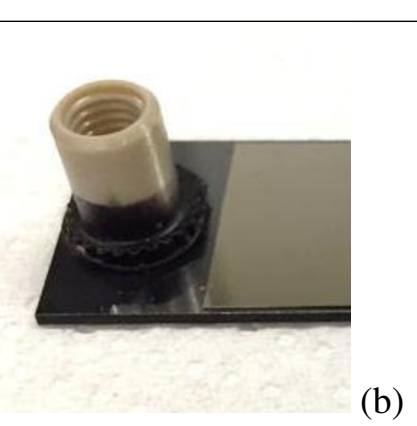

(b)

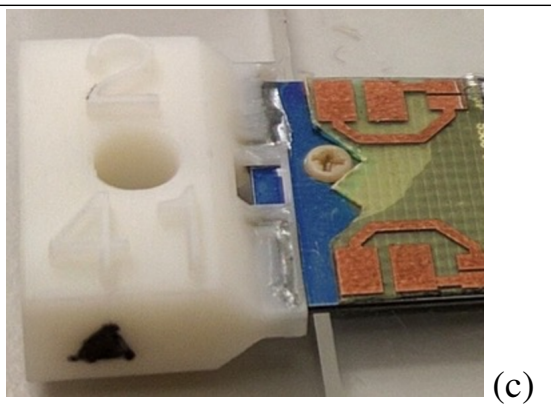

Figure 3: Three solutions for the interface of the micro-channel manifold to the cooling system: (a) the soldered Kovar connectors used by NA62 and LHCb, (b) the PEEK Nanoport connector used for ALICEITS prototyping and (c) the custom 3D-printed connectors developed for sensors with an integrated cooling manifold. Reprinted from Refs. [7, 12].

Kovar, seem to be the most indicated solution. An example of a commercially available connector, the out-of-plane PEEK nanoport connector used in the prototyping of ALICE-ITS structures, is shown in Figure 3 (b). The Bonn-Munich-Valencia group has developed an in-plane connector, that is shown in Figure 3 (c). A custom, 3D-printed plastic connector slides onto the edge of the silicon sensor to connect inlet and outlet. Taking advantage of the tight tolerances on the silicon and connector dimensions, the channels align to a precision of several tens of $\mu \mathrm{m}$ (channel width in these prototype is $350 \mu \mathrm{m}$. Pressure tests of the plastic connectors show they stand over $100 \mathrm{bar}$, well in excess of the requirement for most applications.

\section{Towards fully integrated micro-channel cooling in silicon detectors}

Cooling and detector can be integrated even further by incorporating the micro-channels in the silicon detector itself. The vertex detectors at future lepton colliders present a relatively small power density (typically less than $1 \mathrm{~W} / \mathrm{cm}^{2}$, possibly reduced by a large factor at linear colliders using pulsed powering) and can be operated at room temperature $\left(\sim 20^{\circ} \mathrm{C}\right)$. However, the material budget is extremely tight. Low-pressure, mono-phase micro-channel cooling integrated in the detector may be a good solution in this case.

An SOI process to produce a thin all-silicon ladder with integrated micro-channels was recently demonstrated [12]. The process steps, outlined in Figure 4, represent an extension of the process [16] used to produce the DEPFET active pixel detectors for the Belle II vertex detector $[17,18]$ and linear collider prototypes [19]. The process is fully compatible with the production of depleted silicon sensors.

A small number of mechanical prototypes were produced at HLL. The channels have a relatively large cross section (widths ranging from 200 to $340 \mu \mathrm{m} \times$ a height of $370 \mu \mathrm{m}$ ). The cooling performance of the integrated channels is established by measurements on prototypes. The inlet and outlet are connected to through the connectors of Figure 3 (c). A power of $6 \mathrm{~W}$ is applied on a small area of the sensor of approximately $0.8 \mathrm{~cm}^{2}$, located exactly on top of the cooling channels. Water is pumped though the manifold with a maximum flow rate of $1.5 \mathrm{l} / \mathrm{h}$ and a maximum pressure of less than 6 bar. 
a) oxidation and back side implant of top wafer

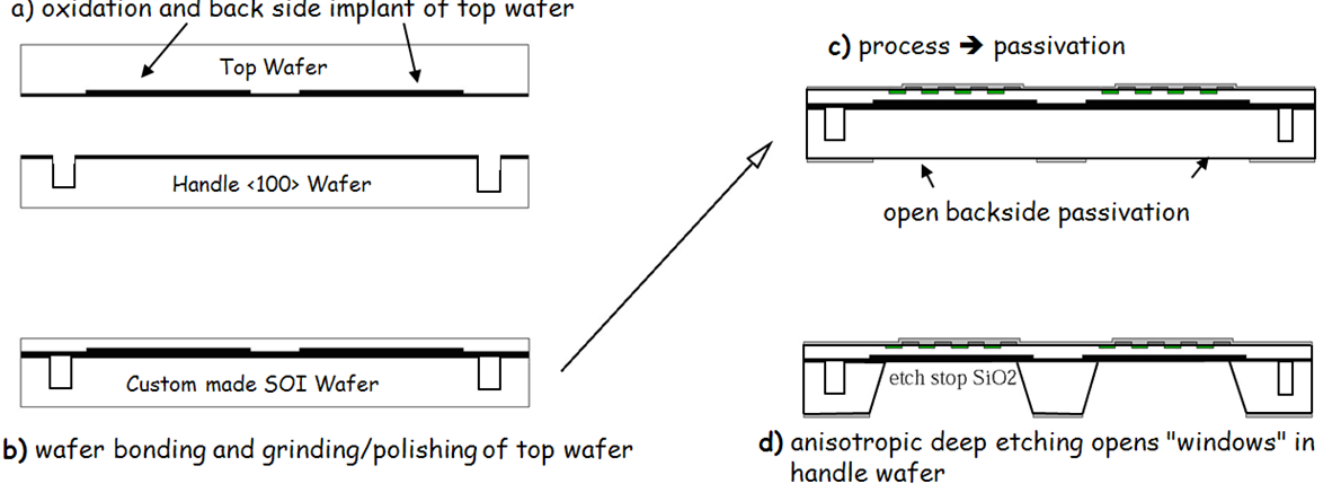

Figure 4: The process sequence for production of thin silicon sensors with electrically active back side implant and integrated cooling channels starts with the oxidation of the top and handle wafer and the backside implantation for the sensor devices; cooling channels are etched into the handle wafer before bonding (a). After direct wafer bonding, the top wafer is thinned and polished to the desired thickness (b). The processing of the devices on the top side of the wafer stack is done on conventional equipment; the openings in the back side passivation define the areas where the bulk of the handle wafer will be removed (c). The bulk of the handle wafer is removed by deep anisotropic wet etching. The etch process stops at the silicon oxide interface between the two wafers (d). The cooling channels are only accessible after dicing of the wafers. Reprinted from Ref. [12].

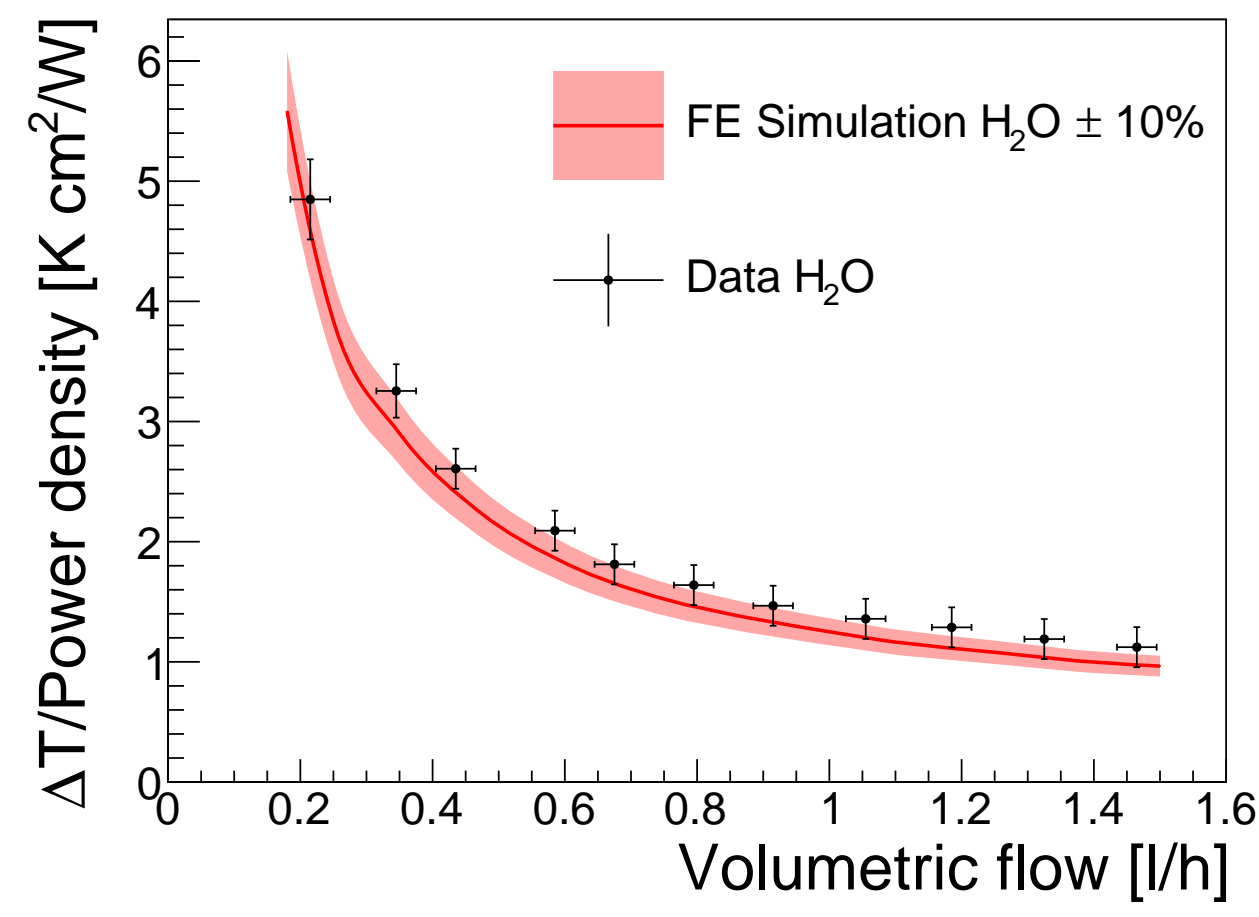

Figure 5: The temperature difference between the hottest point on the sensor surface and the coolant at the inlet - divided by the dissipated power - as a function of the water flow. Reprinted from Ref. [12]. 
In Figure 5 the temperature difference between the hottest point on the sensor surface, divided by the dissipated power, is shown as a function of the volumetric flow. As the increases to $1 \mathrm{l} / \mathrm{h}$, the thermal figure-of-merit approaches 1, exceeding that of classical cooling solutions by a factor five or more. The measurements are reproduced to within $10 \%$ by Finite Element simulations. Static and dynamic distortions are monitored to the $100 \mathrm{~nm}$ level throughout the tests. The liquid flow is not found to have a significant impact on the mechanical stability of the sensor.

\section{Conclusions \& Outlook}

Micro channel cooling is an attractive solution for the cooling of position-sensitive silicon detectors in environments with a limited material budget. Due to the absence of thermal barriers and the close match of the thermal expansion coefficients micro channel cooling offers superior performance compared to more classical cooling strategies. NA62, the first experiment to adopt this technique, has successfully operated a hybrid pixel detector cooled by mono-phase C6F14 flowing through micro-channels in a silicon cooling plate.

The recent extension of the DEPFET all-silicon process to incorporate micro-channel cooling demonstrates the feasibility of a monolithic integration of cooling in the active silicon layer. A thermo-mechanical characterization of a small number of prototypes confirms the potential of this approach. The direct contact of heat source and coolant allows for a thermal figure-of-merit approaching 1, well beyond the performance of existing systems. The liquid flow does not compromise the mechanical stability of the sample.

Micro channel cooling is likely to play an increasingly important role in collider experiments. The first evaporative micro cooling system is being developed for the LHCb VELO upgrade. Groups active in ATLAS, ALICE and future lepton colliders are actively pursuing R\&D in this direction. Technological institutes with close ties with high energy physics can provide in-house processing capacity. Several standard solutions for the connectivity are being developed under the AIDA2020 project.

\section{Acknowledgements}

The results presented in this contribution are the work of a large group of people. The author acknowledges, in particular, the help of L. Andricek (MPG-HLL), L. Eklund (U. Glasgow), N. Flaschel (DESY), M. John (Oxford), A. Mapelli (CERN), C. Marinas (U. Bonn), P. Petagna (CERN), M. Ullan (CNM) and M.A. Villarejo (IFIC Valencia) in the preparation of this report. The reported R \& D has received funding in the the Horizon 2020 Research and Innovation programme of the European Union under Grant Agreement no. 654168. The author acknowledges support from the Spanish national programme for particle physics project FPA2015-65652-C4-3-R (MINECO/FEDER).

\section{References}

[1] G. Romagnoli et al., Silicon micro-fluidic cooling for NA62 GTK pixel detectors, Microelectronic Engineering 145 (2015) 133 - 137. Micro/Nano Devices and Systems 2014 An open focused special thematic issue of Microelectronic Engineering. 
[2] NA62 Collaboration, F. Hahn, F. Ambrosino, A. Ceccucci, H. Danielsson, N. Doble, F. Fantechi, A. Kluge, C. Lazzeroni, M. Lenti, G. Ruggiero, M. Sozzi, P. Valente, and R. Wanke, NA62: Technical Design Document, Tech. Rep. NA62-10-07, CERN, Geneva, Dec, 2010.

[3] M. Fiorini et al., The Gigatracker: An ultra-fast and low-mass silicon pixel detector for the NA62 experiment, Nucl. Instrum. Meth. A628 (2011) 292-295.

[4] GTK working group Collaboration, M. Fiorini, The NA62 GigaTracker, PoS VERTEX2016 (2016).

[5] A. Francescon, A. Mapelli, G. Nuessle, P. Petagna, A. Pezous, P. Renaud, and G. Romagnoli, Application of micro-channel cooling to the local thermal management of detectors electronics for particle physics, Microelectronics Journal 44 (2013), no. 7612 - 618.

[6] NA62 Collaboration, 2016 NA62 Status Report to the CERN SPSC, Tech. Rep. CERN-SPSC-2016-016. SPSC-SR-183, CERN, Geneva, Apr, 2016.

[7] LHCb Collaboration, LHCb VELO Upgrade Technical Design Report, Tech. Rep. CERN-LHCC-2013-021. LHCB-TDR-013, Nov, 2013.

[8] LHCb Collaboration, C. Dean, The LHCb VELO for Phase 1 Upgrade, PoS VERTEX2016 (2016).

[9] CERN PH-DT, VELO Group Collaboration, O. A. de Aguilar Francisco et al., Evaporative $\mathrm{CO}_{2}$ microchannel cooling for the LHCb VELO pixel upgrade, JINST 10 (2015), no. 05 C05014.

[10] ALICE Collaboration, B. Abelev et al., Technical Design Report for the Upgrade of the ALICE Inner Tracking System, Tech. Rep. CERN-LHCC-2013-024. ALICE-TDR-017, Nov, 2013.

[11] N. Flaschel et al., Thermal and hydrodynamic studies for micro-channel cooling for large area silicon sensors in high energy physics experiments, arXiv: 1611.05306.

[12] L. Andricek, M. Boronat, I. Garcia, P. Gomis, C. Marinas, J. Ninkovic, M. P. Rosello, M. A. Villarejo, and M. Vos, Integrated cooling channels in position-sensitive silicon detectors, JINST 11 (2016), no. 06 P06018, [arXiv: 1604.08776 ].

[13] LHCb Collaboration, S. Beole, The upgrade of the ALICE ITS, PoS VERTEX2016 (2016).

[14] A. Francescon, G. Romagnoli, A. Mapelli, P. Petagna, C. Gargiulo, L. Musa, J. R. Thome, and D. D. Col, Development of interconnected silicon micro-evaporators for the on-detector electronics cooling of the future $\{I T S\}$ detector in the $\{A L I C E\}$ experiment at $\{L H C\}$, Applied Thermal Engineering 93 (2016) $1367-1376$.

[15] M. Boscardin, P. Conci, M. Crivellari, S. Ronchin, S. Bettarini, and F. Bosi, Silicon buried channels for pixel detector cooling, Nuclear Instruments and Methods in Physics Research Section A: Accelerators, Spectrometers, Detectors and Associated Equipment 718 (2013) 297 - 298. Proceedings of the 12th Pisa Meeting on Advanced DetectorsLa Biodola, Isola dâĂŹElba, Italy, May 20 âĂŞ 26, 2012.

[16] L. Andricek, G. Lutz, R. H. Richter, and M. Reiche, Processing of ultra-thin silicon sensors for future e+e- linear collider experiments, IEEE Trans.Nucl.Sci. 51 (2004) 1117-1120.

[17] Belle-II Collaboration, T. Abe et al., Belle II Technical Design Report, arXiv: 1011.0352.

[18] DEPFET Collaboration, B. Schwenker, Development and construction of the Belle II DEPFET pixel detector, PoS VERTEX2016 (2016).

[19] DEPFET Collaboration, O. Alonso et al., DEPFET active pixel detectors for a future linear $e^{+} e^{-}$ collider, IEEE Trans.Nucl.Sci. 60 (2010). 